\title{
Preloaded structures for space exploration vehicles
}

\author{
S. Seriani ${ }^{1}$, L. Scalera ${ }^{2}$, A. Gasparetto ${ }^{2}$ and P. Gallina ${ }^{1}$ \\ ${ }^{1}$ Department of Engineering and Architecture, University of Trieste, Trieste, Italy, \\ e-mails:sseriani@units.it; pgallina@units.it. \\ ${ }^{2}$ EnginePolytechnic Department of Engineering and Architecture, University of \\ Udine, Udine, Italy.e-mail: scalera.lorenzo@spes.uniud.it .
}

\begin{abstract}
This paper is devoted to the design and simulation of a rover equipped with 4 preloaded structure legs. Space exploration vehicles (rovers) are employed for moving sensors, transporting planet samples and manipulating small modules. In order to perform such tasks with high accuracy, high mechanical stiffness frames are required. At the same time, a certain degree of compliance is required to mitigate mechanical stress caused by motion over a rough surface or in case of unexpected collisions. For this reasons, preloaded structures could represent a suitable solution. They present sharp stiff-to-compliant transitions at design-determined load thresholds. The paper describes the dynamic model of a single rover leg, the simulation of an impact event and the overall design of system.
\end{abstract}

Keywords: Rover, space exploration, preloaded structures, S-structures, dynamic model.

\section{Introduction}

Space exploration vehicles (rovers) are employed for moving sensors, transporting planet samples and manipulating small modules [1]. In order to perform such tasks with high accuracy, high mechanical stiffness frames are required. At the same time, a certain degree of compliance is required to mitigate mechanical stress caused by motion over a rough surface or in case of unexpected collisions [2]. Preloaded structures can be the effective solution to the problem. These structures are fitted with preloaded springs, in such a way that they exhibit a compliant behavior only when a threshold load is reached [3].

In mechanics, for a single DoF, elasticity is modelled as a linear relation $F=k x$ between force $F$ and deformation $x$, where $k$ is the elastic coefficient. However, for the preloaded structures high stiffness is exhibited up to a certain load, and past that, compliance: this non-linear behaviour (bilinear) is shown in Fig. 1. Indeed, the simplest "soft" linearly elastic compliant link or actuator would have very poor performances, causing oscillations and poor dynamic stability during normal operation. One solution to this problem is applying a preload to 
the relevant elastic components. This arrangement effectively splits the elastic field in two areas, based on loading conditions: one where the system is as rigid as the material which is used for the structure (elastic coefficient $k_{1}$ ) and the other where rigidity is given by the elastic components (coefficient $k_{2}$ ); typically $k_{1} \gg k_{2}$. In the plot, $F_{p}$ is the preload force: indeed, if the applied force $F<F_{p}$, then, in this case, the resulting deformation $\mathrm{x}$ can be less substantial.

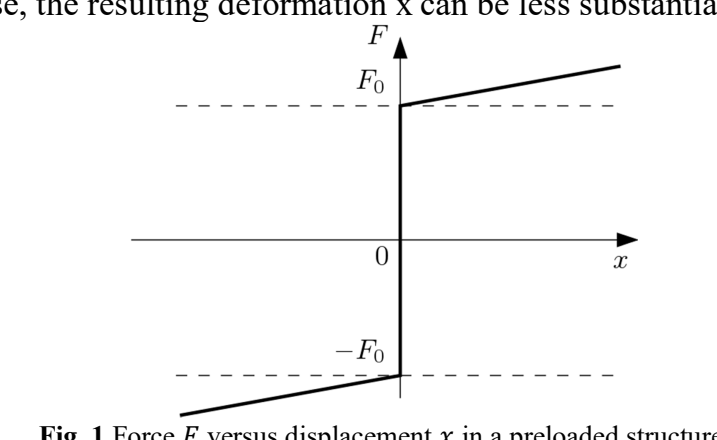

Preloaded structures are employed especially in the field of the collaborative robots (cobots) to keep positional accuracy high, while at the same time allowing for collision mitigation through compliance after a certain force threshold is reached [3]. The use of preload to increase the rigidity of structures can be found also in several areas of structural engineering, e.g. in the design of large telescopes [4], in space docking systems [5] in variable-stiffness mechanisms [6] and even in pipe joints [7]. Usually, these applications were concerned mainly on single-doF systems, such as active or passive joints, link structures etc. In a previous research, we have produced a comprehensive n-DoF dynamic model [8].

In this paper we show the design of a 4 legs rover equipped with 4 independently steering wheels, as shown in Fig. 2. Each leg is made up of a chain of links connected by a series of preloaded mechanisms similar to those described by Medina et al. [9]. In order to verify the dynamic behaviour of the rover during an impact event, a "quarter model" of the rover has been implemented.

In Section 2, the overall design of the rover is presented. Section 3 is devoted to the n-DoF model of the preloaded structures. Simulation results are presented in Section 4.

\section{Planar n-D.o.F. Model}

Let us consider a sequence of $n$ serial links, as visible in Fig. 3 a), each of length $l_{1}, \ldots, l_{n}$, mass $m_{1}, \ldots, m_{n}$, and rotational inertia $J_{1}, \ldots, J_{n}$. A preloaded elastic connection is placed between each contiguous link. The elastic connection can be modeled as a device producing a torque $M\left(\alpha_{i}\right)$, where $\alpha_{i}$ is the relative angle between the $\mathrm{i}^{\text {th }}$ link and the next. The torque $M_{i}\left(\alpha_{i}\right)$ follows a trend similar to that 
shown in Fig. 1 for $F(x)$; however, since this would require discontinuities (splitfield) in the elastic relation, a parametric curve was instead arbitrarily selected to approximate the nominal behaviour, as follows,

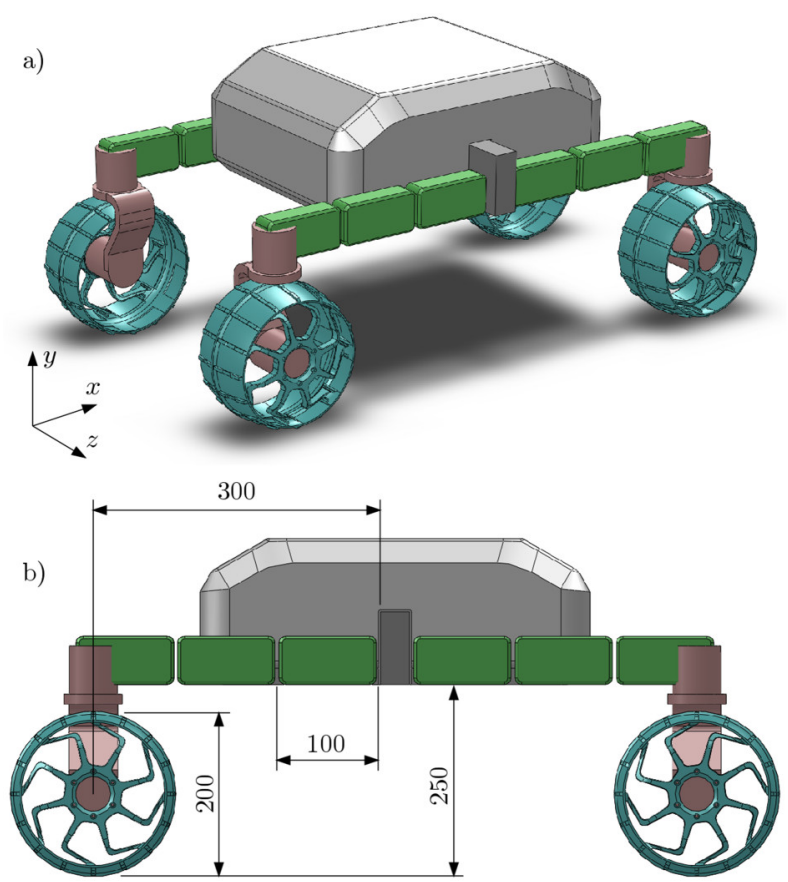

Fig. 2 Design of the rover equipped with 4 preloaded structure legs. In a) an overview is illustrated, whereas in b) the main dimensions are shown in $\mathrm{mm}$.

$M_{i}\left(\alpha_{i}\right) \cong\left(\frac{M_{0, i}{ }^{2}}{\pi}\right) \operatorname{atan}\left(f_{i} \alpha_{i}\right)+k_{i} \alpha_{i}$

where the parameter $M_{0, i}$ represents the preload torque, $k_{i}$ the stiffness of joint and $f_{i}$ is an approximation factor. This type of formulation, being continuous in $\mathbb{C}^{1}$ (as opposed to a split-field one), helps avoiding numerical noise during simulations.

The independent coordinates, in vector form, are $\boldsymbol{q}=\left\{y_{l}, \vartheta_{1}, \alpha_{1}, \ldots, \alpha_{n}\right\}$, where $y_{l}$ and $\vartheta_{1}$ are respectively the vertical position and orientation of the robot chassis. A small-angles approximation is applied to the entire model. The left end-point is constrained horizontally, while it is free to move vertically. This way it is possible to simulate the dynamic behaviour of a single leg (the quarter model of the vehicle). As Fig. $3 \mathrm{~b}$ shows, the wheel is connected to the $4^{\text {th }}$ link and the mass of the rover quarter is concentrated in $\boldsymbol{Q}_{1}$. 


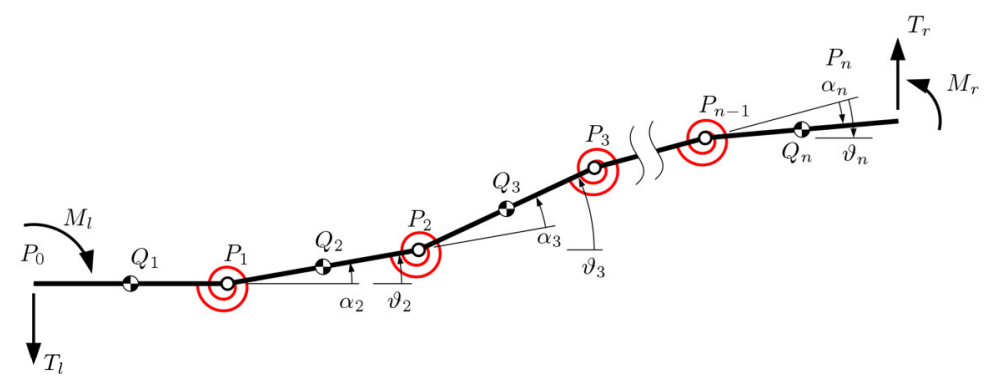

a)

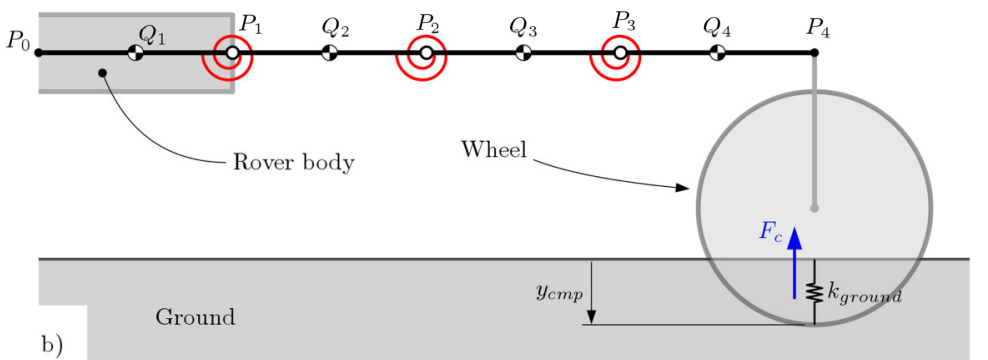

Fig. 3 Diagram of n-DoF preloaded structures. In a) a n-DoF free body diagram is shown as implemented in the general model. In b) a 3-DoF model is shown as implemented in the simulation for the rover limb; indeed the location of the body and of the wheel can be seen as well as the modelization for the contact interface.

From the principle of virtual works, it follows that:

$\delta \Omega+\delta W_{\text {ext }}=0$

where the first term represents the inertial forces virtual work, and the second the external forces virtual work. These can be expanded as follows,

$\delta \Omega=\sum_{i=1}^{n+1} m_{i} \ddot{y}_{i} \delta y_{i}+\sum_{i=1}^{n+1} I_{i} \ddot{\vartheta}_{i} \delta \vartheta_{i}$,

$\delta W_{\text {ext }}=-T_{l} \delta y_{l}-M_{l} \delta \vartheta_{1}+T_{r} \delta y_{r}+M_{r} \delta \vartheta_{n+1}+\sum_{i=1}^{n+1} M\left(\alpha_{i}\right) \delta \alpha_{i}+$ $\sum_{i=1}^{n+1} c_{0} \dot{\alpha}_{i} \delta \alpha_{i}+\sum_{i=1}^{n+1} m g \delta y_{i}$

In matrix form, Eq. (3) can be written as,

$\delta \Omega=\delta \boldsymbol{y}^{T} \boldsymbol{M} \ddot{\boldsymbol{y}}+\boldsymbol{\delta} \boldsymbol{\vartheta}^{T} \mathbf{J} \ddot{\boldsymbol{\vartheta}}$, 
where $\boldsymbol{\delta} \boldsymbol{y}=\left\{\delta y_{1}, \ldots, \delta y_{n}\right\}^{T}$ and $\boldsymbol{\delta} \boldsymbol{\vartheta}=\left\{\delta \vartheta_{1}, \ldots, \delta \vartheta_{n}\right\}^{T}$ are the vector of virtual displacements, $\mathbf{M}$ is the mass matrix, $\boldsymbol{J}$ is the matrix of moments of inertia, $\ddot{\boldsymbol{y}}=\left\{\ddot{y}_{1}, \ldots, \ddot{y}_{n+1}\right\}^{T}$ and $\ddot{\boldsymbol{v}}=\left\{\ddot{\vartheta}_{1}, \ldots, \ddot{\vartheta}_{n+1}\right\}^{T}$ are the vectors of the vertical accelerations of points $y_{1}, \ldots, y_{n+1}$, and of angles $\vartheta_{1}, \ldots, \vartheta_{n+1}$ respectively. Accelerations along the longitudinal axis $\mathrm{x}$ are neglected since small vertical displacements are assumed. In order to reach a formalism based on $\boldsymbol{q}$, some changes in variables are necessary. Indeed, based on simple geometric considerations, we can write,

$$
\left\{\begin{array}{c}
y_{1} \\
y_{2} \\
\vdots \\
y_{n} \\
y_{n+1}
\end{array}\right\} \cong\left[\begin{array}{cccccc}
1 & l / 2 & 0 & \cdots & \cdots & 0 \\
\vdots & l & l / 2 & \ddots & & \vdots \\
\vdots & \vdots & \ddots & \ddots & \ddots & \vdots \\
\vdots & \vdots & & \ddots & l / 2 & 0 \\
1 & l & \cdots & \cdots & l & l / 2
\end{array}\right]\left\{\begin{array}{c}
y_{l} \\
\vartheta_{1} \\
\vdots \\
\vartheta_{n} \\
\vartheta_{n+1}
\end{array}\right\}=\mathbf{A}\left\{\begin{array}{c}
y_{l} \\
\vartheta_{1} \\
\vdots \\
\vartheta_{n} \\
\vartheta_{n+1}
\end{array}\right\}
$$

It is clear that an approximation ( $\sin \vartheta_{i} \cong \vartheta_{i}$ if $\vartheta_{i}$ is small) is applied to get, from the exact formalism, to the linearized one that appears in matrix $\boldsymbol{A}$. Similarly, it can be said that,

$$
\left\{\begin{array}{c}
y_{l} \\
\vartheta_{1} \\
\vdots \\
\vartheta_{n} \\
\vartheta_{n+1}
\end{array}\right\}=\left[\begin{array}{ccccc}
1 & 0 & \cdots & \cdots & 0 \\
0 & 1 & \ddots & & \vdots \\
\vdots & \vdots & \ddots & \ddots & \vdots \\
\vdots & \vdots & & 1 & 0 \\
0 & 1 & \cdots & \cdots & 1
\end{array}\right]\left\{\begin{array}{c}
y_{l} \\
\vartheta_{1} \\
\alpha_{1} \\
\vdots \\
\alpha_{n}
\end{array}\right\}=\mathbf{B} \boldsymbol{q}
$$

It follows immediately that,

$\boldsymbol{y}^{T}=\mathbf{A}$ B $\boldsymbol{q}$,

On a similar note, to write $\boldsymbol{q}$ in terms of the absolute angles $\boldsymbol{\vartheta}$, the following can be written,

$\left\{\begin{array}{c}\vartheta_{1} \\ \vdots \\ \vdots \\ \vartheta_{n+1}\end{array}\right\}=\left[\begin{array}{ccccc}0 & 1 & 0 & \cdots & 0 \\ \vdots & \vdots & \ddots & \ddots & \vdots \\ \vdots & \vdots & & \ddots & 0 \\ 0 & 1 & \cdots & \cdots & 1\end{array}\right] \boldsymbol{q}=\mathbf{C} \boldsymbol{q}$

At this point, Eq. (5) can be written into the following matrix form,

$$
\begin{aligned}
\delta \Omega & =(\mathbf{A} \mathbf{B} \boldsymbol{\delta} \boldsymbol{q})^{T} \mathbf{M} \text { A B } \ddot{\boldsymbol{q}}+(\mathbf{C} \boldsymbol{\delta} \boldsymbol{q})^{T} \mathrm{JC} \ddot{\boldsymbol{q}}= \\
& =\boldsymbol{\delta} \boldsymbol{q}^{T}\left(\mathbf{B}^{T} \mathbf{A}^{T} \mathbf{M A B}+\mathbf{C}^{T} \mathbf{J C}\right) \ddot{\boldsymbol{q}}
\end{aligned}
$$


Regarding the external forces and considering the situation illustrated in Fig. 3, we can write the expression in Eq. (4) as follows,

$\delta W_{\text {ext }}=\left\{\delta y_{l}, \delta \vartheta_{1}, \delta y_{P, r}, \delta \vartheta_{n+1}\right\}\left\{\begin{array}{c}-T_{l} \\ -M_{l} \\ T_{r} \\ M_{r}\end{array}\right\}+\left\{\delta \alpha_{1}, \ldots, \delta \alpha_{n}\right\}\left(\left\{\begin{array}{c}M\left(\alpha_{1}\right) \\ \vdots \\ M\left(\alpha_{n}\right)\end{array}\right\}+\right.$
$\left.c_{o}\left\{\begin{array}{c}\dot{\alpha}_{1} \\ \vdots \\ \dot{\alpha}_{n}\end{array}\right\}\right)+g\left\{\delta y_{1}, \ldots \delta y_{n}\right\}\left\{\begin{array}{c}m_{1} \\ \vdots \\ m_{n}\end{array}\right\}$,

In the above equation, we call $\left\{m_{1}, \ldots, m_{n}\right\}^{T}=\boldsymbol{\mu}$; in order to find the first vector in the first term on the right, we can write,

$$
\left\{\begin{array}{c}
\delta y_{l} \\
\delta \vartheta_{1} \\
\delta y_{P, r} \\
\delta \vartheta_{n+1}
\end{array}\right\}=\left[\begin{array}{llllll}
1 & 0 & 0 & \cdots & 0 & 0 \\
0 & 1 & 0 & \cdots & 0 & 0 \\
1 & l & l & \cdots & l & l \\
0 & 1 & 1 & \cdots & 1 & 1
\end{array}\right] \text { B } \boldsymbol{\alpha} \boldsymbol{q}=\mathbf{D} \boldsymbol{\delta} \boldsymbol{q},
$$

Finally, if $\left\{\alpha_{1}, \ldots, \alpha_{n}\right\}^{T}=\mathbf{E} \boldsymbol{q}$, where $\mathbf{E}$ is obvious, $\boldsymbol{T}_{\text {ext }}=\left\{-T_{l},-M_{l}, T_{r}, M_{r}\right\}^{T}$, and considering Eq. (8) we can write the following matrix form for Eq. (4),

$\delta W_{e x t}=\boldsymbol{\delta} \boldsymbol{q}^{T}\left(\mathbf{D}^{T} \boldsymbol{T}_{\text {ext }}+\mathbf{E}^{T} \bar{M}(\mathbf{E} \boldsymbol{q})+c_{0} \mathbf{E}^{T} \mathbf{E} \dot{\boldsymbol{q}}\right)+(\mathbf{A B})^{\mathrm{T}} \boldsymbol{\mu} g$,

where the $\bar{M}$ operator is defined as $\bar{M}(\mathbf{r})=\left\{M_{1}\left(r_{1}\right), \ldots, M_{n}\left(r_{n}\right)\right\}$, with $\boldsymbol{r}=$ $\left\{r_{1}, \ldots, r_{n}\right\}$ a general vector.

Reassembling Eq. (2) with the expressions in Eq. (10) and (11), it follows that $\left(\mathbf{B}^{T} \mathbf{A}^{T} \mathbf{M A B}+\mathbf{C}^{T} \mathbf{J C}\right) \ddot{\boldsymbol{q}}+c_{0} \mathbf{E}^{T} \mathbf{E} \dot{\mathbf{q}}+\mathbf{E}^{T} \bar{M}(\mathbf{E} \boldsymbol{q})+\mathbf{D}^{T} \boldsymbol{T}_{\text {ext }}+(\mathbf{A B})^{\mathrm{T}} \boldsymbol{\mu} g=0$, thus,

$\mathbf{H} \ddot{\boldsymbol{q}}+\mathbf{S} \dot{\boldsymbol{q}}+\mathbf{G}(\boldsymbol{q})+\mathbf{D}^{T} \boldsymbol{T}_{\text {ext }}+(\mathbf{A B})^{\mathrm{T}} \boldsymbol{\mu} g=0$,

Where $\mathbf{H}$ and $\mathbf{S}$ are the inertial coefficients matrix and the viscous damping coefficient matrix respectively; the term $\mathbf{G}(\boldsymbol{q})$ is the internal forces matrix and models the joints elasticity. It is non-linear with respect to $\boldsymbol{q}$.

Upon impact, considering the wheel stiffness $k_{w}$, the vertical force produced on the structure is

$T_{r}=\left\{\begin{array}{cl}-y_{n+1} k_{w} & \text { if } y_{n+1}<0 \\ 0 & \text { if } y_{n+1} \geq 0\end{array}\right.$

The torque exerted by the wheel is null: $M_{r}=0 . M_{l}$ is the internal force exerted by the rest of the rover on the quarter; since the first link has to remain always 
horizontal, such requirement has been fulfilled by introducing a high stiffness $k_{l}$ and the following boundary condition: $M_{l}=k_{l} \theta_{1}$. Notice that also $T_{l}=0$. The interaction between the ground and the wheel is modeled as a spring system of $k_{\text {ground }}$ stiffness (see Fig. $3 \mathrm{~b}$ ), where the contact force is $F_{c}=k_{\text {ground }} y_{\text {cmp }}$, with $y_{c m p}$ is the ground compenetration measure. In order to produce viable and stable results, the model expressed in Eq. 13 was time-integrated using the classic "RK4" Runge-Kutta method.

\section{Simulation of an impact event}

During deployment, a rover experiences high loads due to impacts with the ground. For this reason, as can be seen on the left in Fig. 4, we defined a use case with a $0.200 \mathrm{~m}$ vertical drop at a gravity level equal to that of earth, which is a conservative approach. Considering that the clearance of the rover is $0.250 \mathrm{~m}$, we elected to limit the deceleration phase to $90 \%$ of that value in order to avoid collision between the ground and the belly of the rover. As the plots in Fig. 4 show, the results of the simulation show the first impact at approximately $t=0.2 \mathrm{~s}$, with a rebound take-off starting at $t=0.56 \mathrm{~s}$, a subsequent free-flight phase that ends with a secondary impact at $t=0.87 \mathrm{~s}$.

\begin{tabular}{lll}
\multicolumn{3}{c}{ Simulation DatA } \\
\hline \multicolumn{2}{l}{ Parameter } & Value \\
\hline$L_{i}$ & {$[\mathrm{~m}]$} & 0.100 \\
$m_{i}$ & {$[\mathrm{~m}]$} & 0.098 \\
$m_{R}$ & {$[\mathrm{Kg}]$} & 1.500 \\
$J_{i}$ & {$\left[\mathrm{Kgm}^{2}\right]$} & $8.2 e-5$ \\
$c_{0}$ & {$[\mathrm{Nm} / \mathrm{s}]$} & 0.300 \\
$y_{l, 0}$ & {$[\mathrm{~m}]$} & 0.200 \\
$k_{\text {ground }}[\mathrm{N} / \mathrm{m}]$ & $1 e 5$ \\
$k_{l}$ & {$[\mathrm{Nm} / \mathrm{rad}]$} & $1 e 6$ \\
$M_{0, i}$ & {$[\mathrm{~N} / \mathrm{m}]$} & {$[4.0 ; 2.7 ; 1.3]$} \\
$f_{i}$ & {$[-]$} & $1 e 4[1.0 ; 0.7 ; 0.3]$ \\
$k_{i}$ & {$[-]$} & 20 \\
$g$ & {$\left[\mathrm{~ms}^{-2}\right]$} & 9.81 \\
\hline
\end{tabular}
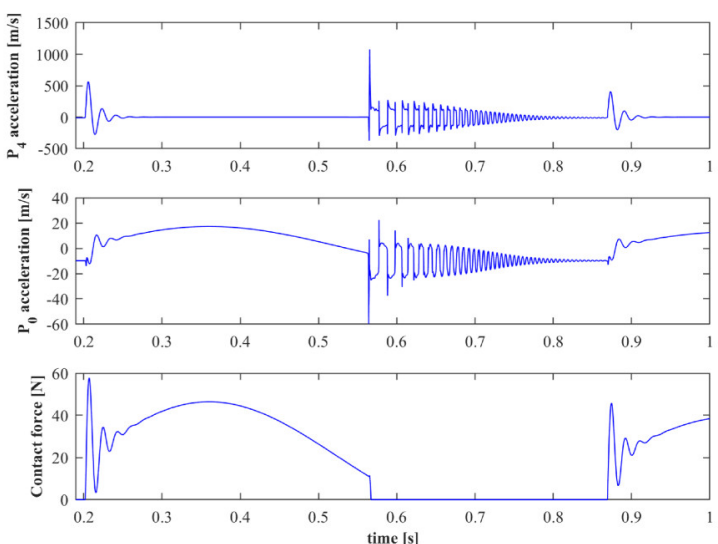

Fig. 4. Impact simulation. On the left, a summary of the main values is reported. On the right, in the top-most plot the acceleration of the wheel joint $P_{4}$ is shown; in the middle, the acceleration can be seen of the body of the rover, corresponding to joint $P_{0}$; the bottom-most chart shows the evolution in time of the contact force between the ground and the wheel.

The lowest point reached by the rover belly is $y=-0.220 \mathrm{~m}$ compared with the standstill value. It is important to note the peak values of the acceleration at point $P_{0}$ and $P_{4}$; indeed, if we recall that the body of the rover is integral to the former, whereas the wheel is connected to the latter, we can appreciate how the rover body experiences lower magnitude accelerations. More specifically, not considering 
low-energy peaks (very small duration), the wheel experiences accelerations in excess of $500 \mathrm{~m} / \mathrm{s}^{2}$, whereas the body sees this value limited to less than $20 \mathrm{~m} / \mathrm{s}^{2}$, sustained, and approximately $60 \mathrm{~m} / \mathrm{s}^{2}$ peak value. As a comparison, these values are close to only 4 times the static force exerted by gravity on the ground. In the top and middle plots of Fig. 4, during the free-flight phase, a strong oscillation can be seen that is highly irregular in nature; this is due to the bi-elastic behavior of the joints. Results show that this type of mechanism can be used effectively to limit accelerations on the body of the rover, while at the same time acting as a stiff structural component when loads are below a certain threshold.

\section{Conclusions}

This paper described the design and the dynamic simulation of a rover for space exploration, equipped with 4 preloaded structure legs. The proposed solution guarantees a high stiffness behavior during conventional operations, as well as a compliance behavior when an expected load (collision or motion over an irregular surface) occurs. At the same time, a certain degree of compliance is required to mitigate mechanical stress caused by motion over a rough surface or in case of unexpected collisions. Simulation results show the benefit introduced by the preloaded structures.

\section{References}

1. S. Seriani, P. Gallina and A. Wedler, "A Modular Cable Robot for Inspection and Light Manipulation on Celestial Bodies", Acta Astronautica, vol. 123, pp. 145-153, 2016.

2. S. Seriani, P. Gallina and A. Wedler, "Dynamics of a Tethered Rover on Rough Terrain", 1st International Conference of IFToMM Italy, IFIT 2016, Advances in Italian Mechanism Science, pp. 355-361, 2016

3. J. López-Martínez, J. L. Blanco-Claraco, D. García-Vallejo and A. Giménez-Fernández, "Design and analysis of a flexible linkage for robot safe operation in collaborative scenarios," Mechanism and Machine Theory, vol. 92, pp. 1-16, 2015.

4. P. Y. Bely, The Design and Construction of Large Optical Telescopes. Astronomy and Astrophysics Library, Springer-Verlag, 2003.

5. L. Olivieri, A. Antonello, L. Savioli and A. Francesconi, "Dynamic behavior of a semiandrogynous small satellite docking interface," in 65th International Astronautical Congress, Toronto, Canada, 2014

6. Y.-S. Wu and C.-C. Lan, "Linear variable-stiffness mechanisms based on preloaded curved beams," Journal of Mechanical Design, vol. 136, 2014.

7. Z. Qin, S. Yan and F. Chu, "Dynamic analysis of clamp band joint system subjected to axial vibration," Journal of Sound and Vibration, vol. 329, pp. 4486-4500, 2010.

8. S. Seriani, L. Scalera, P. Gallina, V. Lughi, "Development of n-DoF preloaded structures for impact mitigation in cobots," Journal of Mechanisms and Robotics, paper accepted, 2018.

9. J. Medina, P. Lozano, A. Jardòn and C. Balaguer, "Design and Characterization of a Novel Mechanism of Multiple Joint," in IEEE/RSJ International Conference on Intelligent Robots and Systems (IROS), Daejeon, Korea, 2016. 